\title{
On the Gers̆gorin Theorem Applied to Radar Polarimetry
}

\author{
E. Lüneburg ${ }^{1}$, A. Danklmayer ${ }^{2}$, and W.-M. Boerner ${ }^{3}$ \\ ${ }^{1}$ EML Consultants, Wessling, Germany \\ ${ }^{2}$ German Aerospace Center, Germany \\ ${ }^{3}$ University of Illinois at Chicago, USA
}

\begin{abstract}
This contribution is concerned with the mathematical formulation and theoretical background of the Gers̆gorin theory in the context of Radar Polarimetry. Named after its founder Semian A. Gers̆gorin the Gers̆gorin theorem basically states that there are certain regions in the complex plane that can be derived from any $n \times n$ complex matrix by rather simple arithmetic operations. These regions are containing more information, specifically its eigenvalues lying within or at the boundaries of circles, where the radii are obtained by the deleted absolute row and/or column sums of the respective $n \times n$ complex matrices.
\end{abstract}

\section{INTRODUCTION}

We consider strict radar backscattering (the monostatic case), characterized by the random Sinclair matrix $S(t)$ in the common linear $\{x, y\}$-basis

$$
S(t)=\left[\begin{array}{ll}
S_{x x}(t) & S_{x y}(t) \\
S_{y x}(t) & S_{y y}(t)
\end{array}\right]
$$

In the case of reciprocal backscattering the Sinclair matrix is symmetric $S_{x y}=S_{y x}$ for a determinitstic or point target and $S_{x y}(t)=S_{y x}(t)$ for any instant of time or space for a reciprocal random target. A change of the orthonormal polarization basis induces a unitary consimilarity transformation for $S(t)$.

$$
S(t) \rightarrow S^{\prime}(t)=U^{T} S(t) U
$$

This implies that the Sinclair matrix $S(t)$ due to its symmetry can be condiagonalized for any instant of time by unitary consimilarity with the unitary matrix $U(t)$. This follows from Takagi's theorem. There is, however, a unique unitary matrix only for point targets with a delta-type probability density function. We consider the backscatter case and omit the subscript. The standard target feature vector in the general case are given by

$$
\vec{k}_{4}(t)=\operatorname{vec} S(t)=\left[\begin{array}{l}
S_{x x}(t) \\
S_{y x}(t) \\
S_{x y}(t) \\
S_{y y}(t)
\end{array}\right]
$$

The corresponding covariance matrices are given by

$$
C_{4}=<\vec{k}(t) \vec{k}_{4}^{\dagger}>
$$

The covariance matrices are Hermitian positive semidefinite and can be diagonalized by general unitary similarity transformations with a certain $4 \times 4$ unitary matrix $V$

$$
\begin{aligned}
& V^{-1} C_{4} V=\Lambda_{4}=\left[\begin{array}{cccc}
\lambda_{1} & 0 & 0 & 0 \\
0 & \lambda_{2} & 0 & 0 \\
0 & 0 & \lambda_{3} & 0 \\
0 & 0 & 0 & \lambda_{4}
\end{array}\right] \text {, } \\
& C_{4}=V \Lambda_{4} V^{-1} \quad \text { with } \quad 0 \leq \lambda_{4} \leq \lambda_{3} \leq \lambda_{2} \leq \lambda_{1} .
\end{aligned}
$$

With $V=\left[\hat{x}_{1}, \hat{x}_{2}, \hat{x}_{3}, \hat{x}_{4}\right]$ we obtain the eigenvalue/eigenvector equations

$$
\begin{aligned}
& C_{4} \hat{x}_{i}=\lambda_{i} \hat{x}_{i} \quad \text { with } \quad<\hat{x}_{i}, \hat{x}_{j}>=\hat{x}_{i}^{\dagger} \hat{x}_{j}=\lambda_{i} \delta_{i j} \\
& (i=1,2,3,4) .
\end{aligned}
$$

All the eigenvectors can be multiplied by arbitrary phase factors $\hat{x}_{i} \rightarrow \exp \left(j \phi_{i}\right) \hat{x}_{i}$. If all four eigenvalues are different there are four one-dimensional $C_{4}$ invariant subspaces: $\operatorname{Span}\left(\hat{x}_{i}\right), i=$ 
$1, \ldots, 4$. The total number of invariant subspaces (including the zero subspace and the entire space $\left.C^{4}\right)$ is $2^{4}=8$. These subspaces assume a particularly simple form if the unitary similarity to the diagonal form $\Lambda_{4}$ is used. Then

$$
\hat{x}_{i}=\hat{e}_{i} \quad \rightarrow \quad \Lambda_{4} \hat{e}_{i}=\lambda_{i} \hat{e}_{i} \quad(i=1, \ldots, 4) .
$$

For backscattering, the space $C^{4}$ containing the general vectors $\vec{k}_{4}(t)$ is restricted to the subspace $C_{s}^{4}$ spanned by the vectors $\vec{k}_{4}^{(s)}(t)$ with $S_{x y}(t)=S_{y x}(t)$. For the covariance matrix this can be expressed in the form

$$
C_{s}^{4}=P C^{4}=\operatorname{Im} P \quad \text { with } \quad P=\left[\begin{array}{cccc}
1 & 0 & 0 & 0 \\
0 & 1 / 2 & 1 / 2 & 0 \\
0 & 1 / 2 & 1 / 2 & 0 \\
0 & 0 & 0 & 1
\end{array}\right]
$$

where $P$ is a projector $P^{2}=P$. The projector $P$ can be expressed in the following way:

$$
P=B^{+} B \quad \text { with } \quad B=\left[\begin{array}{cccc}
1 & 0 & 0 & 0 \\
0 & 1 / \sqrt{2} & 1 / \sqrt{2} & 0 \\
0 & 0 & 0 & 1
\end{array}\right] \quad \text { and } \quad B^{+}=B^{T}=\left[\begin{array}{ccc}
1 & 0 & 0 \\
0 & 1 / \sqrt{2} & 0 \\
0 & 1 / \sqrt{2} & 0 \\
0 & 0 & 1
\end{array}\right]
$$

$B$ is a $3 \times 4$ matrix and hence has no inverse in the ordinary sense, The matrix $B^{+}$is the so-called Moore-Penrose inverse of $B$ and is characterized as the solution of the following equations

$$
B B^{+} B=B \quad \text { and } \quad B^{+} B B^{+}=B^{+} .
$$

Note that $B B^{+}=I_{3}$, the $3 \times 3$ unit matrix.

The operator $B$ is a transformation from $\mathrm{C}^{4} \rightarrow \operatorname{Im} P$ with the properties

$$
\operatorname{Im} B=\left\{\begin{array}{ll}
\operatorname{Im} P & \text { if } \vec{x} \in \operatorname{Im} P \\
0 & \text { if } \vec{x} \in \operatorname{Ker} P
\end{array} .\right.
$$

From the general bi-static scattering matrix $C_{4}$ we obtain for strict backscattering the singular matrix

$$
C_{4 b}=\left[\begin{array}{cccc}
<\left|S_{x x}(t)\right|^{2}> & <S_{x x}(t) S_{x y}^{*}(t)> & <S_{x x}(t) S_{x y}^{*}(t)> & <S_{x x}(t) S_{y y}^{*}(t)> \\
<S_{x y}(t) S_{x x}^{*}(t)> & <\left|S_{x y}(t)\right|^{2}> & <\left|S_{x y}(t)\right|^{2}> & <S_{x y}(t) S_{y y}^{*}(t)> \\
<S_{x y}(t) S_{x x}^{*}(t)> & <\left|S_{x y}(t)\right|^{2}> & <\left|S_{x y}(t)\right|^{2}> & <S_{x y}(t) S_{y y}^{*}(t)> \\
<S_{y y}(t) S_{x x}^{*}(t)> & <S_{y y}(t) S_{x y}^{*}(t)> & <S_{y y}(t) S_{x y}^{*}(t)> & <\left|S_{y y}(t)\right|^{2}>
\end{array}\right] .
$$

This matrix can be decomposed as

$$
\begin{gathered}
C_{4, b}=\operatorname{Re} C_{4, b}+j \operatorname{Im} C_{4, b} \\
\operatorname{Re} C_{4 b}=\left[\begin{array}{cccc}
<\left|S_{x x}(t)\right|^{2}> & \operatorname{Re}<S_{x x}(t) S_{x y}^{*}(t)> & \operatorname{Re}<S_{x x}(t) S_{x y}^{*}(t)> & \operatorname{Re}<S_{x x}(t) S_{y y}^{*}(t)> \\
\operatorname{Re}<S_{x x}(t) S_{x y}^{*}(t)> & <\left|S_{x y}(t)\right|^{2}> & <\left|S_{x y}(t)\right|^{2}> & \operatorname{Re}<S_{x y}(t) S_{y y}^{*}(t)> \\
\operatorname{Re}<S_{x x}(t) S_{x y}^{*}(t)> & <\left|S_{x y}(t)\right|^{2}> & <\left|S_{x y}(t)\right|^{2}> & \operatorname{Re}<S_{x y}(t) S_{y y}^{*}(t)> \\
\operatorname{Re}<S_{x x}(t) S_{y y}^{*}(t)> & \operatorname{Re}<S_{x y}(t) S_{y y}^{*}(t)> & \operatorname{Re}<S_{x y}(t) S_{y y}^{*}(t)> & <\left|S_{y y}(t)\right|^{2}>
\end{array}\right] \\
\operatorname{Im} C_{4 b}= \\
\left.\begin{array}{ccccc}
0 & \operatorname{Im}<S_{x x}(t) S_{x y}^{*}(t)> & \operatorname{Im}<S_{x x}(t) S_{x y}^{*}(t)> & \operatorname{Im}<S_{x x}(t) S_{y y}^{*}(t)> \\
-\operatorname{Im}<S_{x x}(t) S_{x y}^{*}(t)> & 0 & 0 & \operatorname{Im}<S_{x y}(t) S_{y y}^{*}(t)> \\
-\operatorname{Im}<S_{x x}(t) S_{x y}^{*}(t)> & 0 & 0 & \operatorname{Im}<S_{x y}(t) S_{y y}^{*}(t)> \\
-\operatorname{Im}<S_{x x}(t) S_{y y}^{*}(t)> & -\operatorname{Im}<S_{x y}(t) S_{y y}^{*}(t)> & -\operatorname{Im}<S_{x y}(t) S_{y y}^{*}(t)> & 0
\end{array}\right]
\end{gathered}
$$

$\operatorname{Re} C_{4, b}$ is symmetric and $\operatorname{Im} C_{4, b}$ skew-symmetric.

This matrix operator acts in the restricted space $C_{s}^{4}$ which is invariant with respect to the projector $P$. Hence we can write

$$
\begin{aligned}
& C_{4 b}=P C_{4 b} P=B^{+} B C_{4 b} B^{+} B=: B^{+} C_{3} B \\
& \text { with } \quad C_{3}=B C_{4 b} B^{+}
\end{aligned}
$$


or explicitly

$$
C_{3}=\left[\begin{array}{ccc}
<\left|S_{x x}(t)\right|^{2}> & \sqrt{2}<S_{x x}(t) S_{x y}^{*}(t)> & <S_{x x}(t) S_{y y}^{*}(t)> \\
\sqrt{2}<S_{x y}(t) S_{x x}^{*}(t)> & 2<\left|S_{x y}(t)\right|^{2}> & \sqrt{2}<S_{x y}(t) S_{y y}^{*}(t)> \\
<S_{y y}(t) S_{x x}^{*}(t)> & \sqrt{2}<S_{y y}(t) S_{x y}^{*}(t)> & <\left|S_{y y}(t)\right|^{2}>
\end{array}\right] .
$$

Being a similarity transformation the matrices $C_{4 b}$ and $\tilde{C}_{4 b}$ have the same eigenvalues and the matrix $\tilde{C}_{4 b} \mathrm{~h}$ is also Hermitian positive semidefinite. Deflation can be performed in any basis of the target feature vector.

The $3 \times 3$ covariance matrix $C_{3}$ can thus be generated directly from the feature vector

$$
\vec{k}_{3}(t)=B \vec{k}_{3, b}(t)=\left[\begin{array}{c}
S_{x x}(t) \\
\sqrt{2} S_{o}(t) \\
S_{y y}(t)
\end{array}\right] \quad \text { with } \quad S_{o}(t)=S_{x y}(t)=S_{y x}(t) .
$$

by the standard definition

$$
C_{3}=<B \vec{k}_{4, b}(t) \vec{k}_{4, b}^{\dagger}(t) B^{T}>=<\vec{k}_{3}(t) \vec{k}_{3}^{\dagger}(t)>.
$$

The unitary matrix $U^{T} \otimes U^{T}$ has the form

$$
W^{\dagger}=(U \otimes U)^{T}=U^{T} \otimes U^{T}=\left[\begin{array}{llll}
u_{11} u_{11} & u_{11} u_{21} & u_{21} u_{11} & u_{21} u_{21} \\
u_{11} u_{12} & u_{11} u_{22} & u_{21} u_{12} & u_{21} u_{22} \\
u_{12} u_{11} & u_{12} u_{21} & u_{22} u_{11} & u_{22} u_{21} \\
u_{12} u_{12} & u_{12} u_{22} & u_{22} u_{12} & u_{22} u_{22}
\end{array}\right]
$$

and if applied to a vector

$$
\vec{x}=\left[\begin{array}{l}
a \\
b \\
b \\
c
\end{array}\right] \in C_{s}^{4}=P C^{4}=\operatorname{ImP} \Rightarrow \vec{x}^{\prime}=W^{\dagger} \vec{x}=U^{T} \otimes U^{T} \vec{x}=\left[\begin{array}{c}
a^{\prime} \\
b^{\prime} \\
b^{\prime} \\
c^{\prime}
\end{array}\right] \in C_{s}^{4},
$$

This in particular applies to the standard target feature vector $\vec{k}_{4, b}$, i.e., the subspace $C_{s}^{4}$ is invariant under the unitary transformation $U^{T} \otimes U^{T}$.

In general the unitary transformations that diagonalize the covariance matrices are not of the form of a polarimetric basis transformation, i.e., in general

$$
U\left(C_{S}\right) \neq(U(S) \otimes U(S))^{T} \text { and } U\left(C_{J}\right) \neq\left(U\left(C_{J}\right) \otimes U^{*}\left(C_{J}\right)\right)^{T} .
$$

In the following we refer to some results contained in Horn and Johnson [1] and Varga [2]

\section{GERS̆GORIN THEOREM}

Let $\left[a_{i j}\right] \in M_{n}$, and

$$
R_{i}^{\prime}(A) \equiv \sum_{\substack{j=1 \\ j \neq i}}^{n}\left|a_{i j}\right|, 1 \leq i \leq n
$$

denote the deleted absolute row sums of $A$. Then all the eigenvalues of $A$ are located in the union of $n$ discs

$$
U_{i=1}^{n}\left\{z \in C:\left|z-a_{i i}\right| \leq R_{i}^{\prime}(A)\right\} \equiv G(A) .
$$

Furthermore, if a union of $k$ of these $n$ forms a connected region that is disjoint from all the remaining $n-k$ discs then there are precisely $k$ eigenvalues of $A$ in this region.

The region $G(A)$ if often called the Gers̆gorin region (for rows) of $A$; the individual discs in $G(A)$ are called Gers̆gorin discs, and the boundaries of these discs are called Gers̆gorin circles. Since the matrices $A$ and $A^{T}$ have the same eigenvalues, one can obtain a Gers̆gorin disc theorem for columns by applying the Gers̆gorin disc theorem to $A^{T}$ to obtain a region that contains the eigenvalues of $A$ and is specified in terms of deleted absolute column sums

$$
C_{j}^{\prime}(A) \equiv \sum_{\substack{i=1 \\ i \neq j}}^{n}\left|a_{i j}\right|, \quad 1 \leq j \leq n
$$




\section{CONCLUSIONS}

The Gers̆gorin disc theorem is presented and adopted to the covariance matrices used in radar polarimetry, where the theorem shows potential to allow for target identification and classification which has to be further investigated in a follow up contribition.

\section{REFERENCES}

1. Horn, R. A. and C. R. Johnson, Matrix Analysis, Cambridge University Press, New York, 1985.

2. Varga, R. S., Gers̆gorin and his Circles, Springer, 2004.

3. Lüneburg, E. and W.-M. Boerner, "Statistical aspects of radar polarimetry," 43-52 in: P. Russer, M. Mongiardo (Editors), Fields, Networks, Computational Methods, and Systems in Modern Electrodynamics, A Tribute to Leopold B. Felsen (the Proceedings of the Symposium on Fields, Networks and Computation: A Modern View of Engineering Electrodynamics, 2004 June 01-02, Technical University of Munich, Springer-Verlag Berlin-Heidelberg-New York, 2004. 